\title{
ON LOCAL SOLVABILITY OF PSEUDO- DIFFERENTIAL EQUATIONS
}

\author{
A. MENIKOFF
}

\begin{abstract}
A sufficient condition for the local solvability of the equation $u_{t}-A\left(x, t, D_{x}\right) u=f(x, t)$ is proved, where $A$ is a first order pseudo-differential operator with real symbol. This is a special case of the local solvability conjecture of Nirenberg and Treves.
\end{abstract}

Introduction. Let $P(x, D)$ be a linear partial differential operator of principal type with smooth complex value coefficients. The question of when the equation $P u=f$ is locally solvable has been settled by Nirenberg and Treves [4] and Beals and Fefferman [1]. Local solvability is equivalent to the condition:

(P) The imaginary part of $P$ does not change signs on the null bicharacteristics of the real part of $P$.

For a pseudo-differential operator Nirenberg and Treves conjectured that local solvability is equivalent to the condition:

( $\Psi$ ) On every null bicharacteristic of $\operatorname{Re} P$, if $\operatorname{Im} P$ is negative at a point it remains nonpositive from then on.

The purpose of this note is to prove the following special case of this conjecture.

TheOREM 1. Let $P=d / d t-A\left(t, x, D_{x}\right)$ for $(t, x) \in \Omega$ where $A$ is a first order pseudo-differential operator with real symbol $a(t, x, \xi)$. Assume that ( $\Psi)$ if $a\left(t_{0}, x_{0}, \xi_{0}\right)<0$ then for $t>t_{0}, a\left(t, x_{0}, \xi_{0}\right) \leqq 0$; and if $a\left(t_{0}, x_{0}, \xi_{0}\right)=0$ then $\operatorname{grad}_{x, \xi} a\left(t_{0}, x_{0}, \xi_{0}\right)=0$.

Then $P$ is locally solvable.

Theorem 1 is a simple consequence of the following a priori estimate for the adjoint of $P$.

Received by the editors April 9, 1973 and, in revised form, May 4, 1973 and July 2, 1973.

AMS (MOS) subject classifications (1970). Primary 35A05; Secondary 35F05, 35A05.

Key words and phrases. Local solvability, pseudo-differential operators, localization, Gårding's inequality, a priori estimates, Hörmander's partition of unity. 
THEOREM 2. Let $L=d / d t-a\left(t, x, D_{x}\right)$ for $(t, x) \in \Omega=I \times U \subset R^{n+1}$ ( $I$ is an interval containing $t=0$ ) where $A$ is a first order pseudo-differential operator with real symbol $a(t, x, \xi)$. Assume that if $a\left(t_{0}, x_{0}, \xi_{0}\right)>0$ then $a\left(t, x_{0}, \xi_{0}\right) \geqq 0$ for $t>t_{0}$. Also assume that if $a=0$ that $\operatorname{grad}_{x, \xi} a=0$. Then given $\varepsilon>0$, there exists a $\delta$ such that

$$
\|u\|_{0} \leqq \varepsilon\|L u\|_{0} \text { for all } u \in C_{0}^{\infty}((-\delta, \delta) \times U) .
$$

The proof of Theorem 2 follows the lines of the constant coefficient case (cf. Nirenberg-Treves [5]). To do this first we localize $A$ as in Hörmander [2]. We need to prove an estimate similar to the sharp Gårding's inequality, but when the symbol does not have constant sign. Lax-Nirenberg [3] have observed that the positivity of the symbol is needed in the proof of Garrding's inequality only to establish that $\left|\operatorname{grad}_{\xi} a(x, \xi)\right|^{2} \leqq C|a(x, \xi)||\xi|^{-1}$. But this is a consequence of our second assumption about $a$.

Proof of Theorem 2. We use the notation of Hörmander [2]. In particular $a_{\beta}^{\alpha}=\left(i D_{x}\right)^{\beta}\left(i D_{\xi}\right)^{\alpha} a(x, \xi)$.

LemMA 1. If $a(x, \xi)=0$ implies $\operatorname{grad}_{x, \xi} a(x, \xi)=0$ then

$\left|\operatorname{grad}_{\xi} a(x, \xi)\right|^{2}(1+|\xi|)+\left|\operatorname{grad}_{x} a(x, \xi)\right|^{2}(1+|\xi|)^{-1} \leqq C|a(x, \xi)|$.

Proof. If $f(x)=0$ implies $f^{\prime}(x)=0$ then $|f(x)|$ is a nonnegative $C^{2}$ function. Hence $\left|f^{\prime}(x)\right|^{2} \leqq 2|f(x)| \max \left|f^{\prime \prime}\right|$. Applying this inequality to each variable in question and using the pseudo-homogeneity of $a$ we get the lemma.

Hereafter we make the convention that $C$ is any constant depending only on the symbol of $A$.

We now introduce Hörmander's partition of unity. Construct nonnegative functions $\phi_{j}(x) \in C_{0}^{\infty}\left(R^{n}\right)$ such that $\sum_{j=1}^{\infty} \phi_{j}^{2}(x)=1$ and $x, y \in$ supp $\phi_{j}$ implies that $|x-y| \leqq C$, and the supports overlap a bounded number of times. Set $\psi_{j}(\xi)=\phi_{j}\left(\xi|\xi|^{-1 / 2}\right)$ also in $C_{0}^{\infty}\left(R^{n}\right)$. The important properties of the $\psi_{j}$ are that $\sum \psi_{j}^{2}=1$,

$$
\begin{aligned}
& \xi, \eta \in \operatorname{supp} \psi_{j} \text { implies }|\xi-\eta|<C|\xi|^{1 / 2} \text { and } \\
& \sum_{j=1}^{\infty}\left|\psi_{j}(\eta)-\psi_{j}(\xi)\right|^{2} \leqq \frac{C|\xi-\eta|^{2}}{|\xi|}+\frac{8|\xi-\eta|}{|\xi|} .
\end{aligned}
$$

(See Hörmander [2, pp. 141-142] for proofs.) Let $\xi_{j}$ be any point in the support of $\psi_{j}$ and set $u^{j k}(x)=\phi_{k}\left(x\left|\xi_{j}\right|^{1 / 2}\right) \psi_{j}(D) u$ and $\phi_{j k}=\psi_{k}\left(x\left|\xi_{j}\right|^{1 / 2}\right)$. Observe that

$$
\sum_{j, k}\left\|u^{j k}\right\|_{0}^{2}=\|u\|_{0}^{2} .
$$

Choose $x^{j k}$ an arbitrary point in the support of $\phi_{j k}$. 
Lemma 2. Depending on whether $a\left(x^{j k}, \xi^{j}\right) \geqq 0$ or $<0$,

$$
\operatorname{Re}\left(\psi^{j k}(x) \psi_{j}(D) A u, \psi^{j k}(x) \psi_{j}(D) u\right) \geqq R_{j k}(t) \text { or } \leqq R_{j k}(t)
$$

where $\sum_{j, k}\left|R_{j k}(t)\right| \leqq C\|u(t)\|_{0}^{2}$.

Proof OF THeORem 2. Let $L u=f$. By the first assumption on $a$, for each $j, k$ there is a $t_{j k}$ such that $a\left(t, x_{j k}, \xi\right) \leqq 0$ for $t<t_{j k}$ and $\geqq 0$ for $t>t_{j k}$. Let $u \in C_{0}^{\infty}((-\delta, \delta) \times U), \delta$ to be determined in a moment. If $a\left(t, x_{j k}, \xi_{j}\right) \leqq 0$ we have that,

$$
\begin{aligned}
\frac{d\left\|u^{j k}\right\|^{2}}{d t} & =2 \operatorname{Re}\left(\phi^{j k}(x) \psi_{j}(D) u_{t}, u^{j k}\right) \\
& =2 \operatorname{Re}\left(\phi^{j k} \psi_{j}(D)(A u+f), u^{j k}\right) \\
& \leqq 2 R_{j k}+\left\|\phi^{j k}(x) \psi_{j}(D) f\right\|^{2}+\left\|u_{j k}\right\|^{2}
\end{aligned}
$$

Upon integrating the above inequality from $-\delta$ to $t_{j k}$ we get

$$
\left\|u^{j k}(t)\right\|^{2} \leqq \int_{-\delta}^{t_{j k}} 2 R_{j k}(t)+\left\|\phi^{j k} \psi_{j} f\right\|^{2}+\left\|u^{j k}\right\|^{2} d t .
$$

Similarly when $a\left(x^{j k}, \xi_{j}\right) \geqq 0$ we get

$$
\left\|u^{j k}(t)\right\|^{2} \leqq \int_{t_{j k}}^{\delta} 2 R_{j k}(t)+\left\|\phi^{j k} \psi_{j} f\right\|^{2}+\left\|u^{j k}\right\|^{2} d t .
$$

Combining the last two inequalities, summing over $j$ and $k$ and applying Lemma 2 and equation (4) yields that

$$
\begin{aligned}
\|u\|_{0}^{2} & =\sum_{j k}\left\|u^{j k}\right\|^{2} \leqq \int_{-\delta}^{\delta} \sum_{j k} R_{j k}+\left\|\phi^{j k} \psi_{j}(D) f\right\|^{2}+\left\|u^{j k}\right\|^{2} d t \\
& \leqq \int_{-\delta}^{\delta} C\|u\|_{0}^{2}+\|f(t)\|^{2} d t .
\end{aligned}
$$

If we choose $\delta$ small enough so that $4 \delta C<1$ it follows that

$$
\|u(t)\|^{2} \leqq 2 \int_{-\delta}^{\delta}\|f(t)\|^{2} d t
$$

and integrating once more that

$$
\int\|u(t)\|^{2} d t \leqq 2 \delta \int_{-\delta}^{\delta}\|f(t)\|^{2} d t
$$

which proves Theorem 1 .

Proof of Lemma 2. First we will show that,

$$
\sum_{j}\left|\left(\psi_{j}(D) A u, \psi_{j}(D) u\right)-\left(A \psi_{j}(D) u, \psi_{j}(D) u\right)\right| \leqq C\|u\|^{2} .
$$


Let $\hat{a}(\eta, \xi)$ be the Fourier transform of $a(x, \xi)$ with respect to $x$. We may assume that, outside of $U, a$ has compact support in $x$, so it then follows that $|\hat{a}(\eta, \xi)| \leqq C_{N}|\xi|(1+|\eta|)^{-N}$ for any integer $N$. Using Parseval's theorem, the left-hand side of (6) equals

$$
\begin{aligned}
\iint \hat{a}(\eta-\xi, \xi)\left(\phi_{j}^{2}(\eta)\right. & \left.-\psi_{j}(\eta) \psi_{j}(\xi)\right) \hat{u}(\xi) \hat{u}(-\eta) d \xi d \eta \\
& =\frac{1}{2} \iint \hat{a}(\eta-\xi, \xi)\left|\psi_{j}(\eta)-\psi_{j}(\xi)\right|^{2} \hat{u}(\xi) \hat{u}(-\eta) d \xi d \eta .
\end{aligned}
$$

Taking absolute values, summing up over $j$ and applying inequality (3) we may bound the left-hand side of (6) by

$$
\begin{array}{r}
\iint \frac{C_{N}|\xi|}{(1+|\eta-\xi|)^{N}}\left[\frac{C|\eta-\xi|^{2}}{|\xi|}+8 \frac{|\xi-\eta|}{|\xi|}\right]|\hat{u}(\xi)||\hat{u}(\eta)| d \eta d \xi \leqq C\|u\|_{0}^{2} \\
\text { if } N \geqq n+1 .
\end{array}
$$

Next setting $A_{j}=a\left(x, \xi_{j}\right)+\sum_{v=1}^{n} a^{v}\left(x, \xi_{j}\right)\left(D_{v}-\xi_{j}\right)$, we have that

$$
\left|\left(\left(A-A_{j}\right) \psi_{j}(D) u, \psi_{j}(D) u\right)\right| \leqq C\left\|\psi_{j}(D) u\right\|_{0}^{2} .
$$

Parseval's theorem tells us that the left side of (7) equals

$$
\begin{aligned}
& \iint\left\{\hat{a}(\eta-\xi, \xi)-\hat{a}\left(\eta-\xi, \xi_{j}\right)-\sum_{v} \hat{a}^{v}\left(\eta-\xi, \xi_{j}\right)\left(\xi_{v}-\xi_{v}^{j}\right)\right\} \\
& \text { But }\left|\xi-\xi_{j}\right| \leqq C\left|\xi_{j}\right|^{1 / 2} \text { when } \psi_{j}(\xi) \neq 0 .
\end{aligned}
$$

Thus by Taylor's theorem the expression in parentheses is

$$
o\left(\frac{\left|\xi_{j}\right|^{-1}}{1+|\eta-\xi|^{N}}\left|\xi-\xi_{j}\right|^{2}\right)=O\left((1+|\eta-\xi|)^{-N}\right)
$$

and the bound follows. Note that

$$
\begin{aligned}
\operatorname{Re}\left(\phi(x) A_{j} u, \phi u\right) & =\operatorname{Re}\left\{\left(A_{j} \phi u, \phi u\right)+i \int \sum_{v} a^{v} \phi\left(i D_{v}\right) \phi|u|^{2}\right\} \\
& =\operatorname{Re}\left(A_{j} \phi u, \phi u\right) \text { since } a \text { is real. }
\end{aligned}
$$

Combining (6), (7) and (8) we have that

Let

$$
\sum_{j, k}\left|\operatorname{Re}\left(\phi_{j k}(x) \psi_{j}(D) A u, u^{j k}\right)-\operatorname{Re}\left(A_{j} u^{j k}, u^{j k}\right)\right| \leqq C\|u\|_{0}^{2} .
$$

and

$$
u^{j k}(x)=\exp \left\{i\left\langle x, \xi_{j}\right\rangle\right\} v^{j k}\left(\left(x-x^{j k}\right)\left|\xi_{j}\right|^{1 / 2}\right)
$$

$$
\psi_{j}(D) u=\exp \left\{i\left\langle x, \xi_{j}\right\rangle\right\} v^{j}\left(x\left|\xi_{j}\right|^{1 / 2}\right) .
$$


Then $\phi_{k}(x) v^{j}$ and $v^{j k}$ differ only by a translation; $|y| \leqq C$ if $y \in \operatorname{supp} v^{j k}$ and $|\xi| \leqq C$ if $\xi \in \operatorname{supp} v^{j}$. Therefore it follows that

$$
\begin{aligned}
& \int \sum_{k ;|\alpha+\beta| \leqq N}\left|y^{\beta} D^{\alpha} v^{j k}\right|^{2} d y \leqq C \sum_{k ;|\alpha| \leqq N} \int\left|D^{\alpha} v^{j k}\right|^{2} d y \\
& \leqq C \sum_{k ;|\alpha| \leqq N} \int\left|D^{\alpha} \phi_{k}(x) v^{j}(y)\right| d y \\
& \leqq C \sum_{|\alpha| \leqq N} \int\left|D^{\alpha} v^{j}(y)\right| d y \\
& \leqq C_{N}\left|\xi_{j}\right|^{n / 2}\left\|\psi_{i}(D) u\right\|_{0}^{2} .
\end{aligned}
$$

By a change of variables we see that

$$
\begin{aligned}
& \left(A_{j} u^{j k}, u^{j k}\right) \\
& =\left|\xi_{j}\right|^{-n / 2} \int v^{j k}(y)\left\{a\left(x^{j k}+y\left|\xi_{j}\right|^{-1 / 2}, \xi_{j}\right)\right. \\
& \left.\quad+\sum_{v} a^{v}\left(x^{j k}+y\left|\xi_{j}\right|^{-1 / 2}, \xi_{j}\right)\left|\xi_{j}\right|^{1 / 2} D_{v}\right\} v^{j k}(y) d y .
\end{aligned}
$$

By Taylor's theorem, the fact that supp $v^{j k}$ is bounded, and (10), we have that

$$
\begin{aligned}
& \sum_{k}\left(A_{j} u^{j k}, u^{j k}\right)-\left|\xi_{j}\right|^{-n / 2} \int v^{j k}(y)\left\{a\left(x^{j k}, \xi_{j}\right)+\sum_{v} a_{v}\left(x^{j k}, \xi_{j}\right)\left|\xi_{j}\right|^{-1 / 2} y\right. \\
& \left.+\sum_{v} a^{v}\left(x^{j k}, \xi_{j}\right)\left|\xi_{j}\right|^{1 / 2} D_{v}\right\} v^{j k}(y) d y \\
& \leqq\left|\xi_{j}\right|^{-n / 2} \sum_{k,|\beta| \leqq 2 ;|\alpha| \leqq 1} \int\left|y^{\beta} D^{\alpha} v^{j k}\right| d y \leqq C\left\|\psi_{j}(D) u\right\|_{0}^{2} .
\end{aligned}
$$

Finally we have, by using Lemma 1 and the Cauchy-Schwartz inequality and the bound on the support of $v^{j k}$,

$$
\begin{aligned}
\sum_{v} \int a^{v}\left(x^{j k}, \xi^{j}\right)\left|\xi^{j}\right|^{1 / 2} & \left(D_{v}^{\cdot} v^{j k}(y)\right) v^{i k}(y) d y \\
& =\sum_{v} \int \hat{a}^{v}\left(x^{j k}, \xi^{j}\right)\left|\xi^{j}\right|^{1 / 2} \xi_{v}\left|\hat{v}^{i k}(\xi)\right|^{2} d \xi \\
& \leqq \frac{1}{4} \int\left|a\left(x^{j k}, \xi^{j}\right)\right|\left|\hat{v}^{j k}(\xi)\right|^{2} d \xi+\int \frac{C}{\left|\xi_{j}\right|}\left|\xi^{j}\right||\xi|^{2}\left|\hat{v}^{j k}(\xi)\right|^{2} d \xi \\
& \leqq \frac{1}{4} \int\left|a\left(x^{j k}, \xi_{j}\right)\right||v(y)|^{2} d y+\left|\xi_{j}\right|^{n / 2}\left\|u^{j k}\right\|_{0}^{2} .
\end{aligned}
$$


Similarly,

$$
\begin{aligned}
& \sum_{v} a_{v}\left(x^{j k}, \xi^{j}\right)\left|\xi_{j}\right|^{-1 / 2} y\left|v^{j k}(y)\right|^{2} d y \\
& \leqq \frac{1}{4} \int\left|a\left(x^{j k}, \xi^{j}\right)\right|\left|v^{j k}(y)\right|^{2}+\int C y^{2}\left|v^{j k}(y)\right|^{2} d y \\
& \leqq \frac{1}{4}\left|a\left(x^{j k}, \xi^{j}\right)\right|\left|v^{j k}(y)\right|^{2} d y+\left|\xi_{j}\right|^{n / 2}\left\|u^{j k}\right\|_{0}^{2} .
\end{aligned}
$$

If $a\left(x^{j k}, \xi^{j}\right) \geqq 0$ we may combine this with (9) and (11) to get

$$
\left(\phi^{j k}(x) \psi_{j}(D) A u, u^{j k}\right) \geqq-R_{j k}
$$

and $\sum\left|R_{j k}\right| \leqq C|u|_{0}^{2}$. This and similar considerations for the case $a\left(x^{j k}, \xi^{j}\right)<$ 0 completes the proof of the lemma.

\section{REFERENCES}

1. R. Beals and C. Fefferman, On local solvability of linear partial differential equations, Ann. of Math. (2) 97 (1973), 482-498.

2. L. Hörmander, Pseudo-differential operators and non-elliptic boundary value problems, Ann. of Math. (2) 83 (1966), 129-209. MR 38 \#1387.

3. P. Lax and L. Nirenberg, On stability for difference schemes; a sharp Gårding's inequality, Comm. Pure Appl. Math. 19 (1966), 473-492. MR 34 \#6352.

4. L. Nirenberg and F. Treves, On local solvability of linear partial differential equations. I. Necessary conditions, Comm. Pure Appl. Math. 23 (1970), 1-38; II. Sufficient condition, ibid. 459-509; correction; 24 (1971), 279-288. MR 41 \#9064a, b.

5. — Remarks on the solvability of linear equations of evolution, Sympos. Math., VII, Academic Press, New York 1971, pp. 325-338.

Department of Mathematics, Johns Hopkins University, Baltimore, Maryland 21218 